\title{
Modelos de Negocios PARA OMV EN EL ECUADOR
}

\author{
Juan P. Inga $\mathrm{O}^{1, *}$ y Esteban F. Ordoñez $\mathrm{M}^{2}$
}

\section{Resumen}

En el presente trabajo definimos el concepto de un OMV y su situación en el país, se da a conocer el proyecto de ley para este tipo de operador móvil. Se examinan los puntos esenciales que permitirán su ingreso en el Ecuador, posteriormente se definen los modelos de negocios según los niveles de manejo de red en las diferentes formas de interconexión hacia el OMR. A través de este análisis definimos cuál es modelo que más se apega a la realidad del mercado ecuatoriano, entendiendo las características del mercado y los posibles clientes potenciales de un OMV en el Ecuador, por último se revisa la simbiosis entre OMV-OMR-ente regulador, para entender su relación y necesidades. Se realiza este análisis en vista de la eminente entrada de los OMV en el Ecuador.

Palabras clave: MVNE, MVNA, MVNO Business Models, OMV, OMR, PMV.

\begin{abstract}
In this paper we define the concept of an OMV and their situation in the country, has given meet the project bill for this operator mobile network and examines the essential points that allow your entry in Ecuador, subsequently define business models based on the levels of network management of different forms of interconnection to the OMR, through this analysis is defined which is model that conforms to the reality of the ecuadorian market, understanding the characteristics of the market and potential clients an OMV in Ecuador. Finally reviews the symbiosis between OMV-OMR-REGULADOR, to understand their relationship and needs. We carried out this analysis in view ofthe imminent entry of MVNOs in Ecuador.
\end{abstract}

Keywords: MVNE, MVNA, MVNO Business Models, OMV, OMR, PMV.

\footnotetext{
${ }_{1, *}$ Ingeniero electrónico, Docente-Investigador del Grupo de Telecomunicaciones GI-TEL del Centro de Investigaciones Desarrollo e Innovación en Ingenierías CIDII. Carrera de Ingeniería Electrónica, Universidad Politécnica Salesiana, sede Cuenca. Autor para correspondencia jinga@ups.edu.ec

${ }^{2}$ Máster en Desarrollo del Pensamiento, Ingeniero electrónico, Docente-Investigador del Grupo de Telecomunicaciones GI-TEL del Centro de Investigaciones Desarrollo e Innovación en Ingenierías CIDII. Carrera de Ingeniería Electrónica, Universidad Politécnica Salesiana, sede Cuenca. Autor para correspondencia eordonez@ups.edu.ec
}

Recibido: 25-09-2012, Aprobado tras revisión: 15-05-2013.

Forma sugerida de citación: Inga, J. y Ordóñez, E. (2013). "Modelos de negocios para OMV en el Ecuador". InGENIUS. N. ${ }^{\circ}$, (Enero-Junio). pp. 50-56. ISSN: 1390-650X. 


\section{Introducción}

Un OMV u Operador Móvil Virtual (MVNO por sus siglas en inglés Mobile Virtual Network Operator) es un prestador de servicios de telecomunicaciones que ofrece al público el servicio de telefonía móvil y demás servicios móviles avanzados (SMA), permitiendo la transmisión de datos mediante la misma red de su proveedor, conocido como operador móvil de red (OMR o MNO Mobile Network Operator), sin embargo, hay que tomar en cuenta que un OMV puede poseer en su infraestructura, centros de conmutación, transmisión, facturación y recursos de numeración, para poder brindar los mencionados servicios, pero no disponen de red de acceso móvil ni concesión de espectro radio eléctrico [1], por lo que no necesita una inversión tan elevada como los operadores ya establecidos u OMR.

El surgimiento de OMV en la región se debe especialmente a las realidades que se están presentando en el ámbito latinoamericano, estas son: ausencia de nuevos operadores que puedan correr con los gastos que representa la infraestructura de red y toda la logística involucrada para ofrecer SMA y si a esto se le suma el problema de colocar nuevas BS (Base Station) principalmente en regiones densamente pobladas, las posibilidades de un nuevo OMR disminuyen. En la mayoría de los países de la región se encuentra que los servicios móviles poseen un alto nivel de penetración, lo cual reduce de igual forma las posibilidades de ingreso de un nuevo operador.

Según el nivel de integración operacional que se pueda llegar a dar entre un OMR y un OMV se consideran tres niveles o tres tipos de OMV, en el primer nivel se encuentra el OMV completo, que tiene la capacidad de administrar por su propia cuenta la inteligencia de la red móvil, debido a que tiene a su cargo la administración del cerebro de la red, los rangos numéricos y su propio registro de localización, pudiendo realizar interconexiones con los otros OMRs por medio de su OMR anfitrión, con la característica de no disponer del segmento de radio en su acceso de red.

Un OVM completo [1], generalmente dispone en su red de los siguientes puntos principales:

- Red de conmutación de circuitos (CircuitCore).

- Red de conmutación de paquetes (PacketCore).

- Red de operación y mantenimiento (NMS/OSS).

- Red Inteligente (IN).

- Centro de facturación, centro de atención al cliente y plataforma de servicios de valor agregado (VAS).

\footnotetext{
${ }^{1}$ Subscriber Identity Module.
}

- Gestión de datos completa de la tarjeta de usuario $(\mathrm{SIM})^{1}$.

En el segundo nivel tenemos un OMV considerado como revendedor neto de servicios, que simplemente revende un servicio con su propio nombre o marca, dentro de su estructura cuenta tan solo con la parte de marca propia, ventas y marketing [2] y las demás fases de operación son realizadas por un MVNE (Enabler Network Virtual Mobile), el cual ofrece la infraestructura y los servicios necesarios para que el OMV funcione.

Finalmente en el tercer nivel se presentan modelos híbridos de OMV los cuales están constituidos en función del nivel de operación al cual pueden llegar pero no conforman un OMV completo y las fases de operación restantes son realizadas por el MVNE [3], esto será explicado con mayor detalle en secciones subsiguientes.

Es importante analizar los modelos de negocios de los OMV, esto permitirá al lector verificar la necesidad de nuevos operadores móviles en el Ecuador. Además, se verificará que los OMV permiten acceder a nichos de mercado que los operadores tradicionales no toman en cuenta y que a través de la implementación de promociones y marketing adecuado podrían ser explotados.

\section{Las regulaciones en el Ecuador para un OMV}

En nuestro país actualmente se encuentra tratándose un proyecto de ley sobre OMV denominado "Proyecto de reglamento para la prestación del servicio móvil avanzado a través de prestadores móviles virtuales" [4] en el cual constan algunos de los aspectos más generales para la entrada de un OMV en el mercado ecuatoriano, en este proyecto se define a un OMV según su artículo tres, como un prestador móvil virtual (PMV) de servicios finales de telecomunicaciones, de toda transmisión, emisión y recepción de signos, señales, escritos, imágenes, sonidos, voz, datos o información de cualquier naturaleza, utilizando uno de los siguientes esquemas o modalidades:

1. La utilización de infraestructura de un prestador móvil establecido (PME) y de frecuencias esenciales asignadas a un PME para la prestación de servicios móviles avanzados (SMA), sin limitación de utilización de infraestructura propia de un prestador móvil virtual (PMV).

2. La utilización de frecuencias esenciales asignadas a un PME para la prestación del SMA. El PME tiene la obligatoriedad de presentar una oferta básica para la prestación móvil virtual, la misma deberá ser remitida para su aprobación 
e inscripción en la Secretaría Nacional de Telecomunicaciones luego de 45 días de aprobado el mencionado reglamento. Dicha oferta básica deberá contener detalladamente las condiciones técnicas, económicas y legales que permitan la prestación de servicios y el normal funcionamiento por parte del PMV, conforme a sus requerimientos.

Aún falta mucho por hacer en este aspecto, pero lo importante es que se ha comenzado, se ha dado el primer paso. Considerando que en la (Resolución No. 498-25-CONATEL-2002) [5] se propone un régimen de libre competencia, sin prácticas restrictivas, así como los monopolios y a su vez promoviendo la eficiencia, universalidad, accesibilidad, continuidad y la calidad del servicio.

En el artículo 17 [6], numerales 1, 2 y 3 de la Constitución, determina que el Estado fomentará la pluralidad y la diversidad en la comunicación y para el efecto: garantiza el acceso en igualdad de condiciones al uso de las frecuencias del espectro radioeléctrico y a bandas libres para la explotación de redes inalámbricas, precautelando que en su utilización prevalezca el interés colectivo; facilitará el acceso universal a las tecnologías de información y comunicación en especial para las personas y colectividades que carezcan de dicho acceso o lo tengan de forma limitada; y no permitirá el oligopolio o monopolio, directo ni indirecto, de la propiedad de los medios de comunicación y del uso de las frecuencias. El proyecto exige a las operadoras ya establecidas que los OMV puedan tener acceso a sus redes.

La entrada de un OMV, supone que una empresa pueda posicionarse en un nicho de mercado, dando servicios a clientes específicos, aumentando la oferta de servicios de valor agregado, permitiendo nuevos esquemas tarifarios y diferenciándose en el trato al cliente. Se estima que a nivel mundial aproximadamente existen 1122 [7] [8] relaciones establecidas de OMV, tomando en cuenta que de este número $20 \mathrm{OMV}$ aún no están implementados aunque cuentan ya con los permisos de operación.

\section{Modelos de negocios para un OMV}

Se definen dos ámbitos en los modelos de negocios para un OMV. El primero depende de las funciones a ejecutar por el operador virtual en su modelo operativo y el segundo aspecto está relacionado directamente con el cliente. Para realizar un análisis de los modelos es necesario entender las características operativas de un OMV en sus diferentes versiones. En la Figura 1, se indica en primera instancia al OMV en su forma completa, el cual se encarga de gestionar todos los servicios que normalmente están a cargo de un OMR, con la diferencia de que no posee una red de acceso móvil, ni los respectivos permisos por uso de frecuencia. En este punto es en donde un OMV de estas características necesita realizar un convenio de conexión hacia el OMR, el cual estaría encargado de la red de acceso, en este caso el OMV realiza las siguientes tareas [2], [9]:

a) Marca, ventas y marketing: Estas etapas se encuentran definidas a través del posicionamiento de la marca en el mercado, el aseguramiento de los clientes y la red de distribución de los servicios ofrecidos por el OMV.

b) Operaciones: Conformada por el área de atención al cliente y provisión de inventario; incluye los dispositivos de comunicación para el usuario y las tarjetas SIMs. El OMV es quien realiza la logística para poseer sus propias tarjetas con su marca. En esta etapa se fijan los precios y se factura.

c) Contenido y aplicaciones: Consta de la plataforma de servicios de valor agregado SVA y la plataforma de servicios generales.

d) Red: Esta sección posee dos situaciones la parte de los rangos numéricos de los chips y el núcleo de Red.

El segundo tipo de OMV que se muestra en la Figura 1, está constituido solamente por la etapa "a", antes mencionada (marca, ventas y marketing) concibiéndose como un OMV de tipo revendedor. En este caso el OMV puede negociar directamente el acceso con el OMR o realizarlo través de un intermediario denominado MVNE (Mobile Virtual Network Enabler), el MVNE se encargará entonces de las etapas "b, c y d" anteriormente citadas, pudiendo además de realizar la interconexión y negociación con el OMR. El MVNE es quien ofrece los servicios de aplicaciones y contenidos móviles, en este caso el OMV se convierte en un revendedor del MVNE.

Para el último caso que se visualiza en la Figura 1, es similar al caso anterior con la diferencia que ahora, de intermediario se encuentra un MVNA (Mobile Virtual Network Aggregator) este supone una asociación de OMV enlazados a un MVNA. Este aspecto es muy importante debido a que un MVNA puede negociar en mejores términos con el OMR debido a su carácter asociativo de OMVs.

El tercer tipo de OMV está definido como hibrido y viene a ser una mezcla de los dos tipos de OMVs antes mencionados el completo y el revendedor. No se puede definir con precisión para este tipo de OMV las etapas a su cargo, pero puede llegar a manejar desde la etapa "a" hasta la "c", pudiendo incluso manejar puntos intermedios o subetapas. Todo dependerá del servicio que desee brindar así como también del monto a invertir en infraestructura, factores que están 


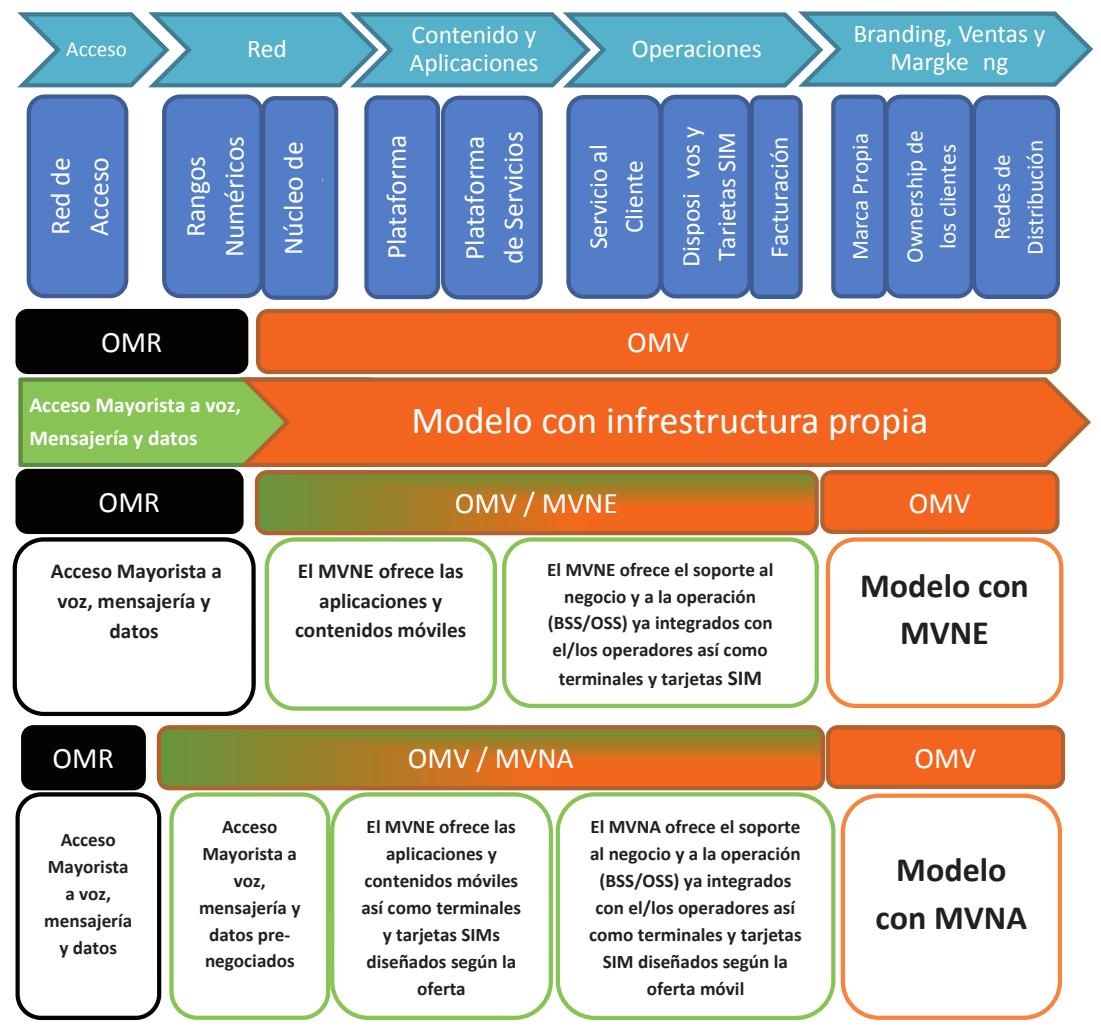

Figura 1. Modelo de un OMV según su interconexión con la OMR [2].

íntimamente relacionados con las características de la empresa que decida ser un OMV híbrido.

De todo lo expuesto, cabe mencionar que un MVNE está concebido para facilitar la infraestructura necesaria para la operación de un OMV, pudiendo llegar a brindar el servicio de interconexión y negociación del host con el OMR anfitrión. Mientras que un MVNA está concebido para la negociación del host incluido los costos por acceso que deberá pagar al OMR anfitrión, adicionalmente brinda el sistema para soporte de negocio y operación. Normalmente los OMV que se encuentran asociación a un MVNA son del tipo revendedores, debido a que estos no tienen un peso significativo para negociar individualmente con el OMR a diferencia que sí negocian en bloque.

Por la situación del mercado móvil del Ecuador y considerando que se va a incursionar por primera vez en este ámbito el modelo de OMV más factible en una primera instancia sería el de un OMV completo, que se encuentre interconectado con el OMR que mayores ventajas competitivas brinde. El mercado ecuatoriano de la telefonía móvil tiene un alto grado de penetración, hoy se estima que en el Ecuador existen más líneas activas que personas $17^{\prime} 357.165^{2}$ líneas a febrero de 2013 [10], por lo que la posibilidad de que en el país existan varios MVNEs y que cada uno de estos posea a la vez varios OMV interconectados no es un panorama que se vea factible, además el número de suscriptores es relativamente pequeño comparado con los mercados de países desarrollados. Están también los altos índices de penetración de SMA 111,67\% [10] y los altos costos iniciales de infraestructura, que permite estimar alrededor de unas tres operadoras de este tipo que subsistan a largo plazo. Para las empresas privadas en el mercado ecuatoriano es complicado posicionarse como un OMV completo, debido a los argumentos antes expuestos, además de los altos costos iniciales que conlleva un OMV de estas características, al posicionamiento de la marca por ello, es más factible que una empresa que ha estado en el ámbito de las telecomunicaciones incursione como OMV, que lo haga una empresa que no ha estado en este campo. Además, están los problemas regulatorios presentes en la constitución, los cuales dan preferencia a las empresas públicas y solamente en caso, de que el estado no pueda brindar dichos servicios se concesionará a la empresa privada. Aunque todos estos problemas sean susceptibles de solución, aún queda la parte de la negociación con el OMR, una sola OMV no tendría el peso necesario como para negociar con el OMR, peor aún, si pretende recién posicionar su marca en el mercado. En este sentido es más factible que existan dos o tres MVNE o OMV completos, que posteriormente, cuando el mercado de operadores virtuales estemás consolidado, podrían aglutinar a OMVs revendedores para brindarles el servicio.

\footnotetext{
${ }^{2}$ Tomando en cuenta terminales de usuario y líneas públicas.
} 
Un OMV está concebido para atender a un nicho o segmentos específicos de mercado, que posiblemente por su situación económica, geográfica no son atendidos adecuadamente por el OMR. Estos segmentos deben ser diversificados para aumentar la rentabilidad del OMV. Mucho dependerá de los contenidos que pueda ofrecer el OMV a sus clientes, uno de estos son las redes sociales junto con aplicaciones multimedia atractivas, interactivas y de fácil acceso, serían una buena receta.

Así los OMV tienen la ventaja de poder enfocarse en microsegmentos desatendidos por parte del OMR, debido a que, a este no le representan un valor significativo, como por ejemplo los jóvenes comprendidos en una edad de 12 a 19 años que no tienen la posibilidad de adquirir un plan pospago. ${ }^{3}$ Los jóvenes son un mercado potencial para un OMV dado que su naturaleza radica en planes prepago. En la Tabla 1, se da a conocer datos sobre el uso de las TIC en el Ecuador que presenta el INEC. Esta información muestra que el uso de los servicios relacionados con internet y redes sociales va tomando fuerza en grupos de edades con menor capacidad adquisitiva como es el grupo de edades de 16 a 24 años, confirmando que es un segmento a tomar en cuenta.

También es un mercado potencial aquellas personas que visitan nuestro país considerado como un destino turístico. Esto significa que gran cantidad de personas ya sea por turismo o por trabajo requieren un sistema prepago de comunicación que sea de rápido acceso y sin muchos trámites a realizar. Además, está el concepto de comunicación entre familiares y amigos o números preferidos que pueden ser un buen segmento a atender y muchos otros segmentos que están en una búsqueda constante de servicios diferenciados y con valor agregado que conlleven a una convergencia "todo IP".

En virtud de esto, el poder mantenerse en el mercado dependerá de la elección e identificación correcta de los segmentos a atacar por parte del OMV, con una buena estrategia empresarial, un atractivo plan tarifario y un buen marketing. Si además se le agrega una buena atención al cliente, existen grandes posibilidades de lograr el éxito a largo plazo con clientes satisfechos y leales a la marca.

Otro aspecto a tomar en cuenta por parte de un OMV es el servicio de roaming (itinerancia), el cual puede ser estructurado de forma tal que permita a un roamer que visita un país determinado obtener costos menores con respecto a los que ofrecen los OMR. Esto se puede lograr a través de acuerdos con OMR de los países vecinos en los que se contemplen precios más bajos, de no ser así, se puede utilizar el servicio de "call back" en el cual una llamada realizada por parte del roamer, a un número determinado en su país de origen es marcada y luego este número retorna la llamada al roamer, con esto se logra bajar costos.

También se puede ofrecer por parte del OMV tarjetas que permiten almacenar múltiples números IMSI para acceder a costos locales para llamadas internacionales [2]. Este aspecto conllevaría inconvenientes en el Ecuador, que a diferencia de otros países, posee regulaciones que no permiten este tipo de comunicación, pues se considerarían un bypass.

Para el servicio de roaming, se debe tener cuidado con la calidad del servicio ofrecido ya que éste depende de la calidad de la señal de los portadores, si estos no poseen un buen sistema de comunicación entre los países, la calidad del servicio se verá degradada.

Dentro del abanico de posibilidades para un OMV, se propone también la posibilidad de comercializar sistemas para rastreo y localización de vehículos, personas u otros artefactos en general, que un determinado usuario requiera. Esto vislumbra un gran mercado potencial ávido de sistemas con estas características, es cierto que empresas como CLARO Y MOVISTAR, ofrecen este tipo de aplicaciones, pero el negocio está en acondicionar los sistemas para las aplicaciones y necesidades personalizadas que den soluciones a usuarios o empresas que requieran sistemas especializados, así el OMV podrá proveer el sistema como tal, a más de brindar el servicio, soporte y mantenimiento.

\subsection{Propuesta de valor de un OMV}

La propuesta de valor de un OMV significa un pilar fundamental para su supervivencia a largo plazo, más aun, en un sector tan dinámico como el de las telecomunicaciones que sufre una metamorfosis tecnológica continuamente. Esto requiere que el OMV tenga la capacidad de estar a la par con los nuevos requerimientos manteniendo compatibilidad tecnológica con el OMR y estar pendiente de las tendencias de sus usuarios, de tal forma de poder reaccionar rápidamente frente a estos cambios.

Las empresas que deseen diversificar su cartera de productos, que ya tienen una marca consolidada y un mercado asegurado, tienen grandes posibilidades de ser un OMV, con la experiencia adquirida, con una infraestructura constituida, pudiesen optar por este nuevo giro de negocios para su mercado actual y posible mercado potencial, pudiendo de esta manera construir una fuente de mercadeo, marketing y publicidad a través de la OMV.

\footnotetext{
${ }^{3}$ Estos datos se confirman en el documento de análisis de la población económicamente activa (PEA) anual presentado por Instituto Nacional de Estadísticas y Censos (INEC) en donde la población de 12 a 19 años representa 10,17\% del PEA para la proyección del 2013.
} 
Tabla 1. Características del mercado de telefonía móvil ecuatoriano [11].

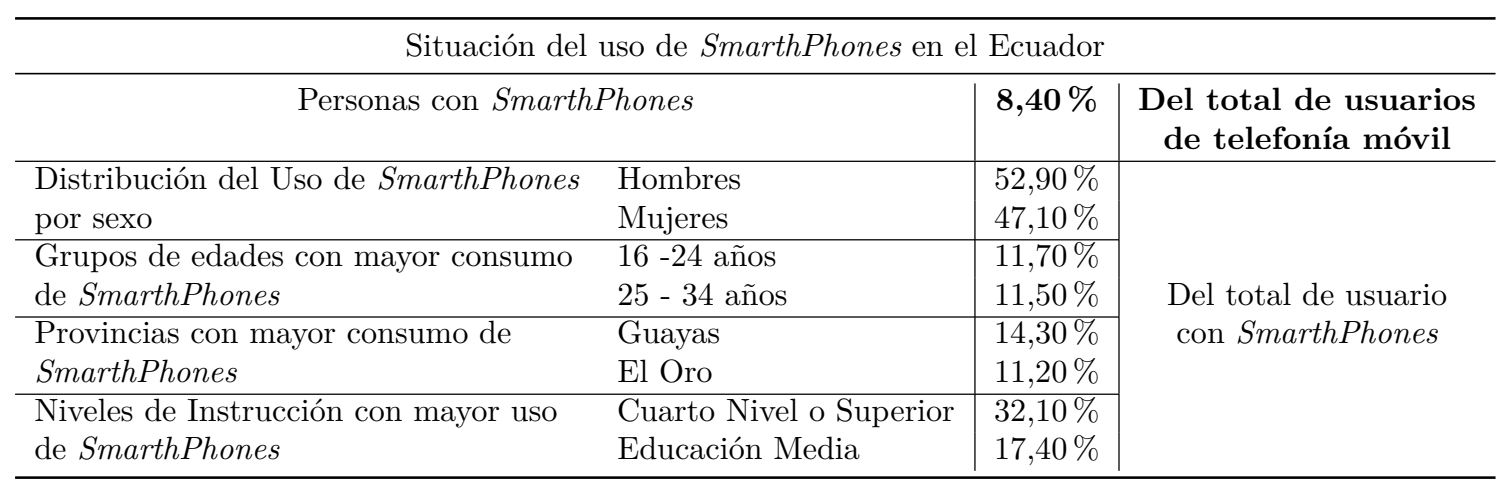

Un OMV, tiene que tener bien claro cuál será su propuesta de valor, la cual puede consistir en brindar una buena y mejor atención, planes y contratos a la medida de los clientes, a mejores precios y sin muchos trámites. Esto se puede lograr revisando las propuestas de valor que poseen los OMR, para determinar cuál es la falencia de estas y construir una propuesta que solvente dichas falencias enfocándose al nicho que se pretende atacar. Se debe tener en cuenta que estos puntos débiles que se observan en un OMR son precisamente por su naturaleza, es decir, al ofrecer servicios masificados no pueden ofrecer demasiados servicios diferenciados.

Existen empresas medianas y pequeñas (PYMES), que tienen necesidades específicas y que serían los potenciales nichos de mercado a ser atendidos por los OMV, ofreciendo servicios específicos tan diferenciados como se posible a determinados mercados. En este sentido una coordinación y logística adecuada entre OMR-OMV son necesarias para brindar servicios de calidad.

\section{Relación OMV-OMR-ente regulador}

Existe una relación triangular entre OMV, OMR y REGULADOR. Por el lado del OMV se encuentran las necesidades de potencializar su marca, aumentar la cartera de clientes en base a la mayor cantidad de servicios a ofrecer. En el caso del OMR, está el interés de aumentar la penetración, mantener a sus clientes, optimizar sus servicios y sacar el mejor provecho a sus activos. Y por último el regulador, sus intereses están en función de lograr un mayor índice de penetración del servicio de telefonía móvil especialmente en los sectores de difícil acceso, disminuir costos para el usuario, incrementar la eficiencia del espectro radio eléctrico que es un bien limitado de un estado, además de disminuir la concentración de mercado en un solo operador, lo cual no es malo, pero hay que precautelar el mal uso de esta posible posición.

El OMV junto con el OMR deben realizar la negociación para la interconexión de sus sistemas, cabe mencionar que deberían ser grupos especializados en el área y multidisciplinarios para lograr el mejor acuerdo posible entre las empresas con características "win-win" [12]. Es decir, en donde ambos actores salgan ganando obteniendo el mejor provecho del acuerdo. Considerando que el OMR por la regulación que se viene y se analizó en el primer apartado de este trabajo, va a estar obligado a interconectarse y permitir el uso de su red por parte del OMV. Esto significa que el OMR deberá planificar su estructura de red de forma que el ingreso del nuevo tipo de operador móvil cumpla con estándares técnicos adecuados para los usuarios propios y los futuros del nuevo operador [13].

Por otro lado el OMV ante la selección de su nicho de mercado y el o los tipos de servicios que desea brindar seleccionará el modelo de negocio más conveniente y al mismo tiempo definirá que entidades de red necesitará para conectarse con su operador anfitrión. Lo ideal es que juntos planifiquen las capacidades de red necesarias para su conexión con el objetivo de asegurar un óptimo servicio para los suscriptores de ambas empresas, más aún sabiendo que el OMR debe considerar una reserva en lo respecta a las capacidades del núcleo de red y de la red de acceso [14].

Por todo esto es primordial que se lleguen a acuerdos entre el OMV y el OMR. En el caso de no lograrse dicho acuerdo, será el ente regulador ${ }^{4}$ quien intervendrá definiendo las reglas del juego como sucede en casos de establecimiento del contrato de interconexión entre dos empresas de telecomunicaciones.

\section{Conclusiones}

Luego de revisar y entender lo que concierne a un OMV, se puede tener un panorama más claro del futuro de las telecomunicaciones en lo que respecta a la

\footnotetext{
${ }^{4}$ La SENATEL como ente regulador intervendrá en caso de que no se llegue a un acuerdo. Según Art. 36 del Reglamento de Interconexión. Resolución 602-29-CONATEL-2006.
} 
telefonía móvil, con un modelo de negocios basado en una elección acertada del segmento de mercado, con un buen plan estratégico y de marketing, buena atención a los clientes, con planes de tarifación atractiva, con propuestas innovadoras de aplicaciones multimedia y servicios de valor agregado, que satisfagan el nicho de mercado elegido por parte de la empresa interesada,es posible establecer un negocio atractivo y que perdure a largo plazo. De tal forma que genere competencia, evolución del negocio e innovación tecnológica. Tomando en cuenta siempre que el área tecnológica cambia continuamente, la empresa debe estar constantemente reestructurándose en sus procesos, para mantenerse a la par de los retos que le imponen las nuevas tecnologías.

Las tendencias en América Latina y especialmente en el Ecuador, apuntan a la apertura por parte de los gobiernos a la creación de reglamentos y regulaciones necesarias para dar paso a los OMV, que permitan la explotación de diversos nichos de mercado sin la necesidad de requerir espectro radio eléctrico, con lo cual se logra una explotación más eficiente del mismo teniendo en cuenta que es un bien no renovable. Así como también un aumento del índice de penetración de SMA. Permitiendo de esta forma la entrada de nuevos actores en el mercado de las telecomunicaciones.

La entrada de los operadores móviles virtuales en el mercado ecuatoriano y en general en cualquier mercado, dependerá del éxito en la relación de negocio entre el OMV y el OMR, para establecer un vínculo de negocios óptimo y adecuado que permita tomar en cuenta el tipo de servicios que el OMV brindará.

\section{Referencias}

[1] M. Ballesteros, "Análisis técnico y regulatorio para la implementación de un operador móvil virtual en el país," Proyecto de graduación, Escuela Politécnica Nacional, Quito, Ecuador, 2009.

[2] D. N. de Planeación de Colombia (DPN), IMOBIX, and I. en Telecomunicaciones (ITC), "Reporte 1. analizar las condiciones técnicas, económicas, de mercado y de impacto de la implementación de operadores móviles virtuales en Colombia. características, conceptos generales y modelos de mercado," Colombia, Programa de apoyo al proceso de participación privada y concesión en infraestructura Tercera Etapa, 2011. [Online]. Available: https://www.dnp.gov.co/LinkClick.aspx? fileticket $=6$ br5UAspLlU\%253D\&tabid $=1255$
[3] M. Jara, "Establecimiento de las condiciones técnicas y regulatorias que permitan el ingreso de operadores móviles virtuales en el mercado de los servicios móviles avanzados del Ecuador," Proyecto de graduación, Escuela Politécnica Nacional, Quito, Ecuador, 2009.

[4] CONATEL, "Proyecto de reglamento para la prestación del servicio móvil avanzado a través de prestadores móviles virtuales," p. 10, 2002. [Online]. Available: http://www.conatel.gob.ec

[5] _ , "Reglamento para la prestación del servicio móvil avanzado," 2002. [Online]. Available: http://www.conatel.gob.ec

[6] Asamblea Constituyente. (2008) Constitución del Ecuador. República del Ecuador. Quito, Ecuador.

[7] Prepaidmvno, 2013. [Online]. Available: www.prepaidmvno.com

[8] Telecompaper, 2013. [Online]. Available: www.telecompaper.com

[9] R. Copeland and N. Crespi, "Modelling MultiMNO business for MVNO in their evolution to LTE, VoLTE \& advanced policy," in Intelligence in Next Generation Networks (ICIN), Octubre 2011, pp. 295-300.

[10] CONATEL. Infraestructura del sector de telefonía móvil SMA - densidad del servicio. Ecuador. [Online]. Available: http://www.conatel.gob.ec/

[11] Instituto Nacional de Estadísticas y Censos (INEC). (2013) Los usuarios de teléfonos inteligentes (smartphone) se incrementaron en un $60 \%$. Ecuador. [Online]. Available: http://www.inec.gob.ec

[12] M. P. De Leon, T. Software, and S. Group, "A user centric always best connected service business model for MVNOs," pp. 1-8, 2010.

[13] R. Copeland and N. Crespi, "Resolving ten MVNO issues with EPS architecture, VoLTE and advanced policy server," in Intelligence in Next Generation Networks (ICIN), October 2011, pp. 29-34.

[14] L. Mastroeni, M. Naldi, and S. Member, "Spectrum reservation option for mobile virtual network operators," in 6th EURO-NF Conference on Next Generation Internet NGI , 2010, pp. 1-8. 